\title{
Relaying Protocols and Delay Analysis for Buffer-aided Wireless Powered Cooperative Communication Networks
}

\author{
Jun Zhan, Xiaohu Tang* and Qingchun Chen \\ School of Information Science and Technology, Southwest Jiaotong University \\ Chengdu, 611756, China \\ [e-mail: zhanjun@my.swjtu.edu.cn,\{xhutang, qcchen\}@swjtu.edu.cn] \\ *Corresponding author: Xiaohu Tang
}

Received December 14, 2017; revised March 7, 2018; accepted April 6, 2018; published August 31, 2018

\begin{abstract}
In this paper, we investigate a buffer-aided wireless powered cooperative communication network (WPCCN), in which the source and relay harvest the energy from a dedicated power beacon via wireless energy transfer, then the source transmits the data to the destination through the relay. Both the source and relay are equipped with an energy buffer to store the harvested energy in the energy transfer stage. In addition, the relay is equipped with a data buffer and can temporarily store the received information. Considering the buffer-aided WPCCN, we propose two buffer-aided relaying protocols, which named as the buffer-aided harvest-then-transmit (HtT) protocol and the buffer-aided joint mode selection and power allocation (JMSPA) protocol, respectively. For the buffer-aided HtT protocol, the time-averaged achievable rate is obtained in closed form. For the buffer-aided JMSPA protocol, the optimal adaptive mode selection scheme and power allocation scheme, which jointly maximize the time-averaged throughput of system, are obtained by employing the Lyapunov optimization theory. Furthermore, we drive the theoretical bounds on the time-averaged achievable rate and time-averaged delay, then present the throughput-delay tradeoff achieved by the joint JMSPA protocol. Simulation results validate the throughput performance gain of the proposed buffer-aided relaying protocols and verify the theoretical analysis.
\end{abstract}

Keywords: Wireless powered, buffer-aided, mode selection and power allocation, throughput-delay tradeoff, Lyapunov optimization.

This work of J. Zhan and X. Tang was supported in part by the National High Technology Research and Development Program of China (863 Program) under Grant 2015AA01A710 and the National Science Foundation of China (NSFC) under Grant 61325005. The work of Q. Chen was supported in part by the NSFC under Grant No. 61771406. 


\section{Introduction}

Recently, radio-frequency wireless energy transfer (RF-WET) technology has got a lot of attention, for the reason that it can provide a stable and continuous energy supply from a RF environment, thus extend the lifetime of energy-constrained wireless networks [1], [2]. The RF-WET technology enables to establish wireless powered communication networks (WPCN), where the wireless communication devices harvest energy from the RF signals to support wireless information transmission [3], [4]. With the aid of RF-WET technology, WPCN avoids the requirement of frequent battery replacement and can be deployed to more communication environments, e.g., Internet of Things (IoT) systems, radio frequency identification (RFID) networks, wireless sensor networks (WSN) [5] and fifth generation (5G) networks [6].

In the current study, there are two typical schemes for implementing WPCN. One scheme is that the communication devices firstly harvest energy from a dedicated power beacon (PB) or access point (AP), then transmit the data to the information receiver by using the harvested energy. In [7] and [8], the optimal time allocation scheme and power-delay tradeoff were presented for a PB-assisted WPCN, respectively. For a multiuser communication network which powered by a AP, the optimal resource allocation techniques which maximize the system throughput were investigated in [9] and [10]. Another typical and widely studied scheme is referred to as the simultaneous wireless information and power transfer (SWIPT), where the RF signals can be utilized for both energy harvesting and information decoding [11]. In order to implement the SWIPT scheme in practice, two receiver designs, named the time splitting (TS) protocol and power splitting (PS) protocol were firstly proposed in [12]. The transmit beamforming design was developed for MISO multiuser systems in [13], [14], where the SWIPT scheme was adopted in each user. The application of SWIPT scheme to a nonorthogonal multiple access (NOMA) network was discussed in [15], in which three user selection schemes were proposed. In addition, as an important application scenario, researches focusing on the cooperative networks with SWIPT have attracted lots of attentions. In [16]-[18], for a three-node relay network, the corresponding ergodic and outage capacities were derived for TS and PS protocols, respectively. In [19], the joint transceiver design and PS ratios optimization at receivers was investigated for a multiuser MISO relay system. Furthermore, the full-duplex wireless powered relay system was explored in [20], [21], where the self-interference signal at the relay can be utilized for energy harvesting, i.e., the so-called self-energy recycling.

Right now, buffer-aided cooperative communication has attracted a growing interest. With the help of data buffer, the received data can be temporarily stored until it is retransmitted to the next node when the channel quality of the corresponding link is better, which has been shown that can improve the performance of cooperative communication networks [22]. Various kinds of buffer-aided relaying schemes, e.g., the adaptive link selection scheme [23]-[25], adaptive transmit mode selection scheme [26]-[28] and relay selection scheme [29]-[31] were widely developed for different buffer-aided relay networks, with the buffer-aided relaying schemes, the throughput performance of cooperative network can be significantly improved. 
Thus far, few researches focused on the wireless powered cooperative communication networks (WPCCN) with energy buffer and data buffer, and less insights were given on the relaying protocol and the impact of buffer on system performance for buffer-aided WPCCN. In particular, in this paper, we consider a buffer-aided WPCCN, in which the source node and relay node are energy constrained, they need to harvest the energy from the dedicated power beacon via wireless energy transfer. Both the source node and relay node are equipped with an energy buffer to store the harvested energy. By using the harvested and stored energy, the source node transmits the data to the destination node via the relay node. In addition, the decode-and-forward relay node is equipped with a data buffer and can temporarily store the received information. Considering the buffer-aided WPCCN, we propose two relaying protocols, namely the buffer-aided harvest-then-transmit $(\mathrm{HtT})$ protocol and buffer-aided joint mode selection and power allocation (JMSPA) protocol, respectively. For the WPCCN with buffer-aided HtT protocol, we obtain the expression of the long-term time-averaged achievable rate of system in closed form. For the buffer-aided JMSPA protocol, maximizing the average achievable rate of WPCCN is formulated as a stochastic optimization problem. According to Lyapunov optimization theory [32], [33], the stochastic optimization problem is transformed into a serious of optimization sub-problems. Based on the channel state information (CSI) and the buffer state information (BSI), these sub-problems are optimally solved by standard convex optimization methods. Then, we obtain the optimal adaptive mode selection scheme, as well as the power allocation strategies for the source node and relay node, respectively. At last, we analyze the theoretical bounds on time-averaged achievable rate, time-averaged data buffer size and time-averaged delay for the buffer-aided JMSPA protocol, and present the throughput-delay tradeoff via theoretical analysis and simulation results.

The rest of the paper is organized as follows. In Section 2, the system model of the buffer-aided WPCCN is introduced. In Section 3, the relaying protocol and the time-averaged achievable rate of the buffer-aided HtT scheme are presented. In Section 4, the buffer-aided JMSPA protocol is investigated, we formulate the optimization problem and provide the optimal solution, then analyze the bounds on time-averaged achievable rate, time-averaged data buffer size and time-averaged delay. Simulation results are provided in Section 5. Finally, Section 6 concludes the paper.

\section{System Model}

As depicted in Fig. 1, we consider a wireless powered cooperative communication network (WPCCN), which consisting of a power beacon (PB), a source node $\mathcal{S}$, a relay node $\mathcal{R}$ and a destination node $\mathcal{D}$. Specially, both the source node and relay node are energy constrained, thus, $\mathcal{S}$ and $\mathcal{R}$ need to harvest energy from the dedicated PB. The PB and all of the nodes in the network are equipped with single antenna, and both of them work at the half-duplex mode, i.e., they cannot transmit and receive simultaneously. In addition, there is no direct link between $\mathcal{S}$ and $\mathcal{D}$, i.e., the source communicates with the destination only via the relay [16]-[18]. 


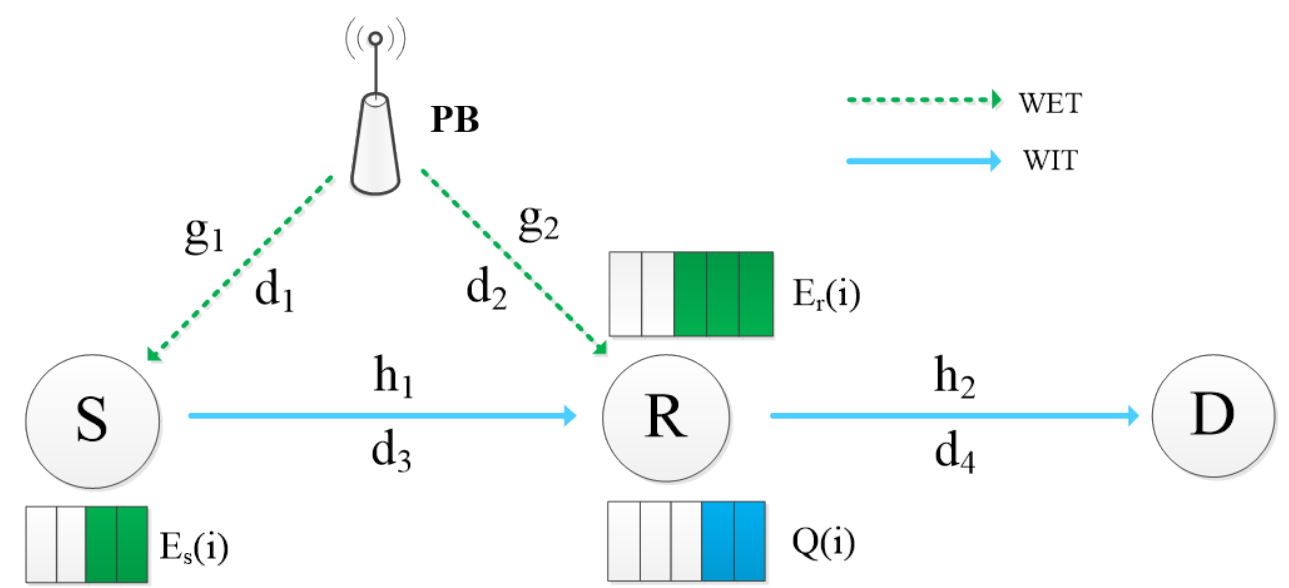

Fig. 1. Buffer-aided wireless powered cooperative communication network.

Time is divided into slots of equal length $T$. In time slot $i$, the gains of the channel from the PB to $\mathcal{S}$ and $\mathcal{R}$ are denoted by $g_{1}(i)$ and $g_{2}(i)$, the channel gains of the $\mathcal{S}-\mathcal{R}$ link and $\mathcal{R}-\mathcal{D}$ link are denoted by $h_{1}(i)$ and $h_{2}(i)$, respectively. Specifically, we assume that the channels are under block fading, i.e., the channel gains keep constant for the duration of one time slot and vary independently from one slot to the next.

$\mathcal{S}$ is equipped with an energy buffer, $\mathcal{R}$ is equipped with an energy buffer and a data buffer. In time slot $i$, the energy buffer state of $\mathcal{S}$ is denoted by $E_{s}(i)$, the energy buffer state and data buffer state of $\mathcal{R}$ are denoted by $E_{r}(i)$ and $Q(i)$, respectively. In order to simplify the analysis, the size of all the buffers are assumed to be infinite, therefore the buffer would not be overflowed. For the WPCCN, there are two transmission stages, named wireless energy transmission (WET) stage and wireless information transmission (WIT) stage, respectively. During the WET stage, the PB broadcasts the energy signal to $\mathcal{S}$ and $\mathcal{R}$, then $\mathcal{S}$ and $\mathcal{R}$ harvest energy with the received signal and store the harvested energy in their energy buffer, respectively. During the WIT stage, we assume that the source node always has data to transmit. In the $\mathcal{S}-\mathcal{R}$ link WIT stage, $\mathcal{S}$ transmits signal to $\mathcal{R}$ by using the stored energy. The received signal at $\mathcal{R}$ will be decoded and temporarily stored in the data buffer until it is transmitted to $\mathcal{D}$ by $\mathcal{R}$ with the stored energy in the $\mathcal{R}-\mathcal{D}$ link WIT stage.

In practical application, this model can be adopted in a wireless sensor network, which is deployed for forest fire detection, water quality monitoring or land slide detection, and so on. In these application scenarios, the random distributed WET-enabled sensor nodes are powered by a dedicated power beacon. On the other hand, for the purpose of alleviating the near-far effect, the near sensor node can be acted as a relay to help the far sensor node transmit the collected data to the data receiver. Furthermore, in order to utilize the harvested energy more efficiently, it is necessary to equip an energy buffer for the sensor nodes. 


\section{Throughput Analysis of The Buffer-aided Harvest-then-Transmit Relaying Protocol}

In this section, the buffer-aided harvest-then-transmit protocol is investigated. In the following, the buffer-aided HtT relaying scheme is introduced firstly, then the time-averaged achievable rate of the buffer-aided HtT scheme is derived.

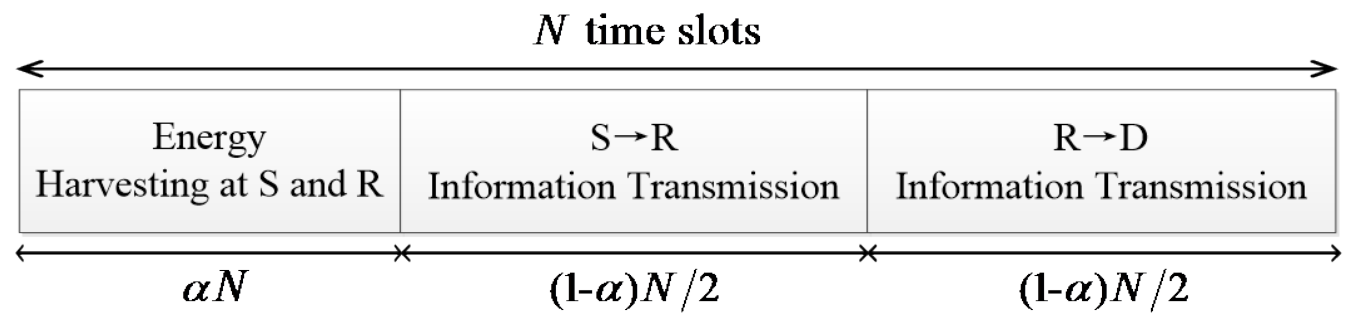

Fig. 2. Buffer-aided Harvest-then-Transmit relaying scheme.

The harvest-then-transmit relaying protocol has been developed for different WPCCNs without buffer in [7], [9], [34], where the WET stage and the WIT stage are implemented within one time-slot. Different from these previous works, we introduce energy buffer and data buffer to WPCCN, in a buffer-aided relay network, with the aid of a data buffer, the relay node is allowed to receive for a number of time slots before retransmitting the received information to destination node, which has been proved that can improve the throughput performance of relay network [35]. Motivated by this result, considering the WPCCN with energy buffer and data buffer in Fig. 1, we propose a buffer-aided HtT scheme, which is shown in Fig. 2, where $N$ is the number of consecutive transmission time slots and $\alpha \in[0,1]$ is the fraction of time slots in which $\mathcal{S}$ and $\mathcal{R}$ harvest energy from the $\mathrm{PB}$. Then, the buffer-aided HtT relaying protocol is described as follows: The first $\alpha N$ time slots are allocated to the WET stage for the PB to broadcast energy signal to $\mathcal{S}$ and $\mathcal{R}$; The remaining $(1-\alpha) N$ time slots are divided into two parts of equal length, which are assigned to the information transmission of the $\mathcal{S}-\mathcal{R}$ link and $\mathcal{R}-\mathcal{D}$ link, respectively.

\subsection{WET Stage}

In time slot $i \in\{1,2, \cdots, \alpha N\}$, the energy signal broadcasted by the $\mathrm{PB}$ is $x_{e}$, and $\mathbb{E}\left\{\left|x_{e}\right|^{2}\right\}=1$. The transmit power of the PB is set to be a constant, denoted by $P$. Then, in time slot $\boldsymbol{i}$, the received signal at $\mathcal{S}$ and $\mathcal{R}$ can be formulated, respectively, as

$$
y_{s}^{e}(i)=\frac{1}{\sqrt{d_{1}^{m}}} \sqrt{P} g_{1}(i) x_{e}+n_{s}^{e}(i)
$$

and

$$
y_{r}^{e}(i)=\frac{1}{\sqrt{d_{2}^{m}}} \sqrt{P} g_{2}(i) x_{e}+n_{r}^{e}(i)
$$

where $d_{1}$ and $d_{2}$ are the distance between the PB with $\mathcal{S}$ and $\mathcal{R}$ respectively, $m$ is the path loss coefficient, $\boldsymbol{n}_{\boldsymbol{s}}^{e}(\boldsymbol{i})$ and $\boldsymbol{n}_{r}^{e}(\boldsymbol{i})$ are the received energy noise signals at the source node and relay node, respectively. 
Comparing to the power of energy signal $x_{e}$, the power of the energy noise signal is sufficiently small such that the energy harvested by the noise signal can be ignored. Thus, in time slot $i$, the collected energy of $\mathcal{S}$ and $\mathcal{R}$ can be calculated, respectively, as

$$
e_{h s}(i)=\frac{\eta P\left|g_{1}(i)\right|^{2}}{d_{1}^{m}} T \quad \text { and } \quad e_{h r}(i)=\frac{\eta P\left|g_{2}(i)\right|^{2}}{d_{2}^{m}} T
$$

where $\eta \in(0,1]$ denotes the energy conversion efficiency.

Hence, at the end of the WET stage, the harvested and stored energy of $\mathcal{S}$ and $\mathcal{R}$ can be represented, respectively, as

$$
E_{h s}=\sum_{i=1}^{\alpha N} e_{h s}(i)=\sum_{i=1}^{\alpha N} \frac{\eta P\left|g_{1}(i)\right|^{2}}{d_{1}^{m}} T
$$

and

$$
E_{h r}=\sum_{i=1}^{\alpha N} e_{h r}(i)=\sum_{i=1}^{\alpha N} \frac{\eta P\left|g_{2}(i)\right|^{2}}{d_{2}^{m}} T
$$

\section{2 $\mathcal{S}-\mathcal{R}$ Link WIT Stage}

In time slot $i \in\{\alpha N+1, \alpha N+2, \cdots,(1+\alpha) N / 2\}$, the source transmits information to the relay with the stored energy. The average transmit power of $\mathcal{S}$ is

$$
P_{s}(i)=\lim _{N \rightarrow \infty} \frac{E_{h s}}{(1-\alpha) N T / 2}=\frac{2 \alpha \eta P \mathbb{E}\left[\left|g_{1}(i)\right|^{2}\right]}{(1-\alpha) d_{1}^{m}}
$$

The signal-to-noise ratio (SNR) at the relay node is expressed as

$$
\gamma_{R}(i)=\frac{P_{s}(i)\left|h_{1}(i)\right|^{2}}{d_{3}^{m} \sigma_{r}^{2}}
$$

where $d_{3}$ is the distance of the $\mathcal{S}-\mathcal{R}$ link, $n_{r}(i)$ is the additive circular symmetric complex gaussian white noise at the relay node, and $n_{r}(i) \sim \mathcal{C N}\left(0, \sigma_{r}^{2}\right)$.

As a result, in the first half of the WIT stage, the stored information bits in the data buffer of $\mathcal{R}$ can be written as

$$
\tau_{1}=\sum_{i=\alpha N+1}^{(1+\alpha) N / 2} T \log _{2}\left(1+\gamma_{R}(i)\right)=\sum_{i=\alpha N+1}^{(1+\alpha) N / 2} T \log _{2}\left(1+\frac{P_{S}(i)\left|h_{1}(i)\right|^{2}}{d_{3}^{m} \sigma_{r}^{2}}\right)
$$

\section{3 $\mathcal{R}-\mathcal{D}$ Link WIT Stage}

In time slot $i \in\{(1+\alpha) N / 2+1,(1+\alpha) N / 2+2, \cdots, N\}$, the relay forwards the stored information to the destination with the collected energy. The average transmit power of $\mathcal{R}$ is given by

$$
P_{r}(i)=\lim _{N \rightarrow \infty} \frac{E_{h r}}{(1-\alpha) N T / 2}=\frac{2 \alpha \eta P \mathbb{E}\left[\left|g_{2}(i)\right|^{2}\right]}{(1-\alpha) d_{2}^{m}}
$$

Similarly, the signal-to-noise ratio at the destination node is expressed as 


$$
\gamma_{D}(i)=\frac{P_{r}(i)\left|h_{2}(i)\right|^{2}}{d_{4}^{m} \sigma_{d}^{2}}
$$

where $d_{4}$ is the distance of the $\mathcal{R}-\mathcal{D}$ link, $n_{d}(i)$ is the additive circular symmetric complex gaussian white noise at the relay node, and $n_{d}(i) \sim \mathcal{C N}\left(0, \sigma_{d}^{2}\right)$.

Hence, the number of bits sent from $\mathcal{R}$ to $\mathcal{D}$ in the second half of the WIT stage can be written as

$$
\tau_{2}=\sum_{i=(1+\alpha) N / 2+1}^{N} T \log _{2}\left(1+\gamma_{D}(i)\right)=\sum_{i=(1+\alpha) N / 2+1}^{N} T \log _{2}\left(1+\frac{P_{r}(i)\left|h_{2}(i)\right|^{2}}{d_{4}^{m} \sigma_{d}^{2}}\right)
$$

Therefore, the time-averaged achievable rate of the buffer-aided HtT relaying scheme is given by

$$
\begin{aligned}
\tau_{H t T} & =\min \left\{\lim _{N \rightarrow \infty} \frac{1}{N T} \tau_{1}, \lim _{N \rightarrow \infty} \frac{1}{N T} \tau_{2}\right\} \\
& =\frac{1-\alpha}{2} \min \left\{\mathbb{E}\left[\log _{2}\left(1+\frac{2 \alpha \eta P \mathbb{E}\left[\left|g_{1}(i)\right|^{2}\right]\left|h_{1}(i)\right|^{2}}{(1-\alpha) d_{1}^{m} d_{3}^{m} \sigma_{r}^{2}}\right)\right], \mathbb{E}\left[\log _{2}\left(1+\frac{2 \alpha \eta P \mathbb{E}\left[\left|g_{2}(i)\right|^{2}\right]\left|h_{2}(i)\right|^{2}}{(1-\alpha) d_{2}^{m} d_{4}^{m} \sigma_{d}^{2}}\right)\right]\right\}
\end{aligned}
$$

\section{Buffer-aided Joint Mode Selection and Power Allocation Relaying Protocol}

Unlike the fixed time slot allocation policy in the buffer-aided harvest-then-transmit protocol, in this section, a dynamic time slot allocation relaying protocol is investigated. For the considered buffer-aided WPCCN, as $\mathcal{S}$ is equipped with energy buffer and $\mathcal{R}$ is equipped with both data buffer and energy buffer, then each time slot can be allocated to WET stage for energy harvesting or to WIT stage for information transmission. Based on this fact, we define three transmission modes, namely the WET-EH mode, the WIT-SR mode and the WIT-RD mode, which correspond to the WET stage, the $\mathcal{S}-\mathcal{R}$ link WIT stage and the $\mathcal{R}-\mathcal{D}$ link WIT stage, respectively. Then, we present the problem formulation for maximizing the time-averaged achievable throughput of the buffer-aided WPCCN with joint mode selection and power allocation (JMSPA) and derive the optimal buffer-aided JMSPA relaying protocol.

Our proposed buffer-aided JMSPA protocol can be implemented in two manners, namely the distributed manner and the centralized manner, which have been discussed in [36] for buffer-aided relay selection policies. In this paper, for the buffer-aided WPCCN, we consider the centralized implementation, where the central node is employed to make a decision which includes information regarding which transmission mode is selected and the power allocation schemes for $\mathcal{S}$ and $\mathcal{R}$. To this end, the central node has to acquire the buffer state information (BSI) of all buffers and the channel state information (CSI) of all links in each time slot. The CSI acquisition of each link can be realized by channel estimation, which needs to choose the appropriate pilot sequences and channel estimation methods. On the other hand, $\mathcal{S}$ and $\mathcal{R}$ know their own buffer states, by designing a feedback mechanism, all the obtained BSIs and CSIs can be gathered at the central node and used to design the buffer-aided JMSPA protocol. 
Since we focus on the CSI/BSI-based adaptive relaying protocols and the achievable performance of the considered buffer-aided WPCCN, for the sake of simplicity, we assume that the central node can acquire ideal CSI/BSI in each time slot. Based on this assumption, we can obtain the optimal joint mode selection and power allocation scheme and determine the optimal throughput performance our protocol can achieve.

\subsection{Buffer Dynamics and Problem Formulation}

We use the binary decision variables $C_{k}(i) \in\{0,1\}, k \in\{1,2,3\}$ to indicate which transmission mode is adopted in time slot $i$. More specifically, we have

$$
\begin{cases}c_{1}(i)=1, & \text { If the WET-EH mode is selected, } \\ c_{2}(i)=1, & \text { If the WIT-SR mode is selected, } \\ c_{3}(i)=1, & \text { If the WIT-RD mode is selected. }\end{cases}
$$

Since the PB and all nodes work on the half-duplex mode, thus only one of the three modes is selected for transmission in each time slot. Hence, we have $c_{1}(i)+c_{2}(i)+c_{3}(i)=1$ for arbitrary time slot $i$.

With the binary mode selection variables, in time slot $i$, the energy buffer queue $E_{s}(i)$ of $\mathcal{S}$ and the energy buffer queue $E_{r}(i)$ of $\mathcal{R}$ update as following

$$
\begin{aligned}
& E_{s}(i+1)=E_{s}(i)+e_{a s}(i)-e_{d s}(i) \\
& E_{r}(i+1)=E_{r}(i)+e_{a r}(i)-e_{d r}(i)
\end{aligned}
$$

where $e_{a s}(i)=c_{1}(i) \eta P\left|g_{1}(i)\right|^{2} T / d_{1}^{m}$ and $e_{d s}(i)=c_{2}(i) P_{s}(i) T$ are the harvested and consumed energy of $\mathcal{S}$ in time slot $i$, respectively. $e_{a r}(i)=c_{1}(i) \eta P\left|g_{2}(i)\right|^{2} T / d_{2}^{m}$ and $e_{d r}(i)=c_{3}(i) P_{r}(i) T$ are the harvested and consumed energy of $\mathcal{R}$ in time slot $i$, respectively.

The data buffer queue $Q(i)$ of $\mathcal{R}$ updates as

$$
Q(i+1)=\max [Q(i)-r(i), 0]+s(i)
$$

where $s(i)=c_{2}(i) T \log _{2}\left(1+\frac{P_{s}(i)\left|h_{1}(i)\right|^{2}}{d_{3}^{m} \sigma_{r}^{2}}\right)$ is the number of bits sent from $\mathcal{S}$ to $\mathcal{R}$, and $r(i)=c_{3}(i) T \log _{2}\left(1+\frac{P_{r}(i)\left|h_{2}(i)\right|^{2}}{d_{4}^{m} \sigma_{d}^{2}}\right)$ is the number of bits sent from $\mathcal{R}$ to $\mathcal{D}$.

Considering $N$ time slots, the long-term time-averaged achievable throughput of the buffer-aided WPCCN is given by

$$
\tau=\lim _{N \rightarrow \infty} \frac{1}{N} \sum_{i=1}^{N} s(i)
$$

In order to maximize the time-averaged achievable throughput, we investigate the optimal adaptive mode selection scheme, as well as the optimal transmit power allocation scheme for $\mathcal{S}$ and $\mathcal{R}$. By jointly optimizing the mode selection variables $c_{1}(i), c_{2}(i), c_{3}(i)$ and the powers $P_{s}(i), P_{r}(i)$, the time-averaged achievable throughput maximization problem can be 
formulated for $N \rightarrow \infty$ :

$$
\begin{array}{ll}
\mathcal{P} 1: \max _{\substack{c_{k}(i), P_{s}(i), P_{r}(i)}} & \lim _{N \rightarrow \infty} \frac{1}{N} \sum_{i=1}^{N} s(i) \\
\text { subject to } & C 1: \lim _{N \rightarrow \infty} \frac{1}{N} \sum_{i=1}^{N} s(i) \leq \lim _{N \rightarrow \infty} \frac{1}{N} \sum_{i=1}^{N} r(i), \\
& C 2: \lim _{N \rightarrow \infty} \frac{1}{N} \sum_{i=1}^{N} e_{d s}(i) \leq \lim _{N \rightarrow \infty} \frac{1}{N} \sum_{i=1}^{N} e_{a s}(i), \\
& C 3: \lim _{N \rightarrow \infty} \frac{1}{N} \sum_{i=1}^{N} e_{d r}(i) \leq \lim _{N \rightarrow \infty} \frac{1}{N} \sum_{i=1}^{N} e_{a r}(i), \\
& C 4: 0 \leq e_{d s}(i) \leq E_{s}(i), \quad \forall i \\
& C 5: 0 \leq e_{d r}(i) \leq E_{r}(i), \quad \forall i \\
& C 6: c_{k}(i)\left[c_{k}(i)-1\right]=0, \quad k \in\{1,2,3\}, \forall i \\
& C 7: c_{1}(i)+c_{2}(i)+c_{3}(i)=1, \quad \forall i .
\end{array}
$$

- Constraint C1 means that the time-averaged arrival rate to the data buffer queue of $\mathcal{R}$ is no more than the time-averaged transmission rate out, thus ensures the stability of the data buffer queue.

- Constraints C2 and C3 denote that the time-averaged consumed energy would not exceed the time-averaged harvested energy for $\mathcal{S}$ and $\mathcal{R}$, respectively.

- Constraints C4 and C5 stand for the energy consumption restriction for $\mathcal{S}$ and $\mathcal{R}$ in each time slot, i.e., the consumed energy does not exceed the stored energy in the energy buffer of $\mathcal{S}$ and $\mathcal{R}$, respectively.

- Constraints C6 and C7 ensure that $c_{k}(i)$ can only be 0 or $1, k \in\{1,2,3\}$, and only one transmission mode is selected for transmission in each time slot.

\subsection{Problem Transformation through Lyapunov Optimization Theory}

Due to the binary variables $c_{k}(i)$ and the non-linear and non-convex constrains C1-C7, the optimization problem $\mathcal{P} 1$ is a non-convex mixed integer non-linear program (MINLP) problem, which cannot be solved by convex optimization methods given in [37]. In general, as the CSI and BSI are known in advance, the problem $\mathcal{P} 1$ can be optimally solved with exhaustive search method. However, this method suffers from a high computational complexity, which increases exponentially with the number of time slots $N$, and is denoted by $3^{N}$. Consequently, this method cannot be implemented in practice, especially for large $N$. Motivated by the previous works in this field [8], [33], we solve the optimization problem $\mathcal{P} 1$ via Lyapunov optimization method, as it develops a low complexity algorithm to achieve the mode selection and power allocation scheme, while revealing the throughput-delay tradeoff in buffer-aided WPCCN.

Let $\mathbf{Z}(i)=\left[Q(i), E_{s}(i), E_{r}(i)\right]$ denotes the vector of current buffer queue, and define a Lyapunov function $L(i)$ as follows:

$$
L(i)=\frac{1}{2} Q(i)^{2}+\frac{\mu_{1}}{2}\left[\phi_{1}-E_{s}(i)\right]^{2}+\frac{\mu_{2}}{2}\left[\phi_{2}-E_{r}(i)\right]^{2}
$$


where $\mu_{1}$ and $\mu_{2}$ are positive constants, $\phi_{1}$ and $\phi_{2}$ are perturbation values for the energy buffer queue of $\mathcal{S}$ and $\mathcal{R}$, respectively.

The change of Lyapunov function over one time slot can be represented by Lyapunov drift $L(i+1)-L(i)$. The one-slot conditional Lyapunov drift can be defined as

$$
\Delta(i)=\mathbb{E}\{L(i+1)-L(i) \mid \mathbf{Z}(i)\}
$$

where the expectation $\mathbb{E}\{\cdot\}$ is with respect to the randomness of CSI and BSI. The objective of our joint mode selection and power allocation scheme is to maximize the time-averaged achievable throughput, while stabilizing the data buffer queue of $\mathcal{R}$. By minimizing the conditional Lyapunov drift $\Delta(i)$, the backlogs at data buffer can be reduced to a lower level, which keeps the stability of data buffer queue. At the moment, the second part of our objective is solved. In order to solve the first part, i.e., achieving a large time-averaged achievable rate, we construct the drift-plus-penalty function as follows

$$
\Delta(i)-V \mathbb{E}\{s(i) \mid \mathbf{Z}(i)\}
$$

where $V>0$ is a system control parameter that affects the tradeoff between the backlog of data buffer and the time-averaged achievable throughput.

Regarding the drift-plus-penalty function, we have the following lemma:

Lemma 1: In time slot $i$, the drift-plus-penalty function is upper bounded by

$$
\begin{aligned}
& \Delta(i)-V \mathbb{E}\{s(i) \mid \mathbf{Z}(i)\} \leq B+Q(i) \mathbb{E}[(s(i)-r(i)) \mid \mathbf{Z}(i)]-V \mathbb{E}\{s(i) \mid \mathbf{Z}(i)\} \\
& \quad+\mu_{1}\left[\phi_{1}-E_{s}(i)\right] \mathbb{E}\left[\left(e_{d s}(i)-e_{a s}(i)\right) \mid \mathbf{Z}(i)\right]+\mu_{2}\left[\phi_{2}-E_{r}(i)\right] \mathbb{E}\left[\left(e_{d r}(i)-e_{a r}(i)\right) \mid \mathbf{Z}(i)\right]
\end{aligned}
$$

where $B$ is a finite constant, and satisfies

$$
\begin{aligned}
B \geq & \frac{\mathbb{E}\left[\left(s(i)^{2}+r(i)^{2}\right) \mid \mathbf{Z}(i)\right]}{2}+\frac{\mu_{1} \mathbb{E}\left[\left(e_{a s}(i)-e_{d s}(i)\right)^{2} \mid \mathbf{Z}(i)\right]}{2} \\
& +\frac{\mu_{2} \mathbb{E}\left[\left(e_{a r}(i)-e_{d r}(i)\right)^{2} \mid \mathbf{Z}(i)\right]}{2}
\end{aligned}
$$

The proof is similar to [8, Theorem 1], for minimizing the conditional Lyapunov drift $\Delta(i)$ to stabilize the buffer queue and maximizing the average achievable throughput, we need to minimize the right side of (22) in Lemma 1 by joint mode selection and power allocation. Based on the Lyapunov optimization theory, the problem of minimizing the right side of (22) can be turned into a series of sub-problems within each time slot, and given by

$\mathcal{P} 2$ :

$$
\min _{\substack{c_{1}(i), c_{2}(i), c_{3}(i), P_{s}(i), P_{r}(i)}}
$$

$$
\begin{aligned}
& Q(i)[s(i)-r(i)]-V s(i)+\mu_{1}\left[\phi_{1}-E_{s}(i)\right]\left[e_{d s}(i)-e_{a s}(i)\right] \\
& \quad+\mu_{2}\left[\phi_{2}-E_{r}(i)\right]\left[e_{d r}(i)-e_{a r}(i)\right]
\end{aligned}
$$

\section{subject to}

$$
C 4, C 5, C 6, C 7 \text { in (18) }
$$

At the moment, we have transformed the stochastic optimization problem $\mathcal{P} 1$ into a deterministic optimization problem $\mathcal{P} 2$ by using Lyapunov optimization theory. In the next subsection, we solve the problem $\mathcal{P} 2$ by employing the standard convex optimization methods and drive the joint mode selection and power allocation scheme within each time slot.

\subsection{Joint Mode Selection and Power Allocation in Each Time Slot}

Since $c_{1}(i)+c_{2}(i)+c_{3}(i)=1$, and the value of $c_{k}(i), k \in\{1,2,3\}$ must be 0 or 1 , there are only three cases, i.e., $c_{1}(i)=1, c_{2}(i)=0, c_{3}(i)=0 ; c_{1}(i)=0, c_{2}(i)=1, c_{3}(i)=0$ and 
$c_{1}(i)=0, c_{2}(i)=0, c_{3}(i)=1$. In the next, we will analyze the three cases and derive the joint optimal mode selection and power allocation policy.

When $c_{1}(i)=1, c_{2}(i)=0, c_{3}(i)=0$, the WET-EH mode is selected for transmission. In this case, we have $e_{a s}(i)=\frac{\eta P\left|g_{1}(i)\right|^{2}}{d_{1}^{m}} T, e_{d s}(i)=0, e_{a r}(i)=\frac{\eta P\left|g_{2}(i)\right|^{2}}{d_{2}^{m}} T, e_{d r}(i)=0$, $s(i)=0$ and $r(i)=0$. Then, the value of the objective function of optimization problem $\mathcal{P} 2$ is denoted by

$$
F_{1}^{*}=\mu_{1}\left[E_{s}(i)-\phi_{1}\right] \frac{\eta P\left|g_{1}(i)\right|^{2}}{d_{1}^{m}} T+\mu_{2}\left[E_{r}(i)-\phi_{2}\right] \frac{\eta P\left|g_{2}(i)\right|^{2}}{d_{2}^{m}} T
$$

When $c_{1}(i)=0, c_{2}(i)=1, c_{3}(i)=0$, the WIT-SR mode is selected for transmission. In this situation, we have $e_{a s}(i)=0, e_{d s}(i)=P_{s}(i) T, e_{a r}(i)=0, e_{d r}(i)=0, r(i)=0$ and $s(i)=T \log _{2}\left(1+\frac{P_{s}(i)\left|h_{1}(i)\right|^{2}}{d_{3}^{m} \sigma_{r}^{2}}\right)$. Then, the optimization problem $\mathcal{P} 2$ can be reformulated as

$$
\begin{aligned}
\mathcal{P} 3: \quad \min _{P_{s}(i)} & {[Q(i)-V] T \log _{2}\left(1+\frac{P_{s}(i)\left|h_{1}(i)\right|^{2}}{d_{3}^{m} \sigma_{r}^{2}}\right)+\mu_{1}\left[\phi_{1}-E_{s}(i)\right] P_{s}(i) T } \\
\text { subject to } & 0 \leq P_{s}(i) T \leq E_{s}(i)
\end{aligned}
$$

Solving problem $\mathcal{P} 3$, we can obtain

Theorem 1: The optimal transmit power of $\mathcal{S}$ is given by

$$
P_{s}^{*}(i)= \begin{cases}0, & \text { if } \begin{array}{r}
Q(i) \geq V, \phi_{1} \geq E_{s}(i) \\
\cup Q(i)>V, \Delta \geq 0 \\
\frac{E_{s}(i)}{T},
\end{array} \\
\text { if } \begin{array}{r}
Q(i)<V, \phi_{1}<E_{s}(i) \\
\cup Q(i)>V, \Delta<0
\end{array} \\
{\left[\frac{[Q(i)-V]}{\mu_{1} \ln 2\left[E_{s}(i)-\phi_{1}\right]}-\frac{d_{3}^{m} \sigma_{r}^{2}}{\left|h_{1}(i)\right|^{2}}\right]^{\dagger},} & \text { otherwise }\end{cases}
$$

where $[x]^{\dagger}=\min \left\{\max [x, 0], \frac{E_{s}(i)}{T}\right\}$, and $\Delta$ is given as bellow

$$
\Delta=\mu_{1}\left[\phi_{1}-E_{s}(i)\right] E_{s}(i)+[Q(i)-V] \log _{2}\left(1+\frac{E_{s}(i)\left|h_{1}(i)\right|^{2}}{d_{3}^{m} \sigma_{r}^{2} T}\right) T
$$

Proof: Please refer to Appendix A for the proof.

When the transmit power of $\mathcal{S}$ is set to be $P_{s}^{*}(i)$, the minimal value of the objective function of optimization problem $\mathcal{P} 3$ is achieved and denoted by $F_{2}^{*}$.

Eq. (27) indicates that the allocated transmit power of $\mathcal{S}$ depends on the CSI of the $\mathcal{S}-\mathcal{R}$ link, the energy buffer state of $\mathcal{S}$ and the data buffer state of $\mathcal{R}$.

When $c_{1}(i)=0, c_{2}(i)=0, c_{3}(i)=1$, the WIT-RD mode is selected for transmission. Similarly, we have $e_{a s}(i)=0, e_{d s}(i)=0, e_{a r}(i)=0, e_{d r}(i)=P_{r}(i) T, s(i)=0$, 
$r(i)=T \log _{2}\left(1+\frac{P_{r}(i)\left|h_{2}(i)\right|^{2}}{d_{4}^{m} \sigma_{d}^{2}}\right)$. Then, the optimization problem $\mathcal{P} 2$ can be reformulated as

$$
\begin{aligned}
\mathcal{P} 4: \quad \min _{P_{r}(i)} & -Q(i) T \log _{2}\left(1+\frac{P_{r}(i)\left|h_{2}(i)\right|^{2}}{d_{4}^{m} \sigma_{d}^{2}}\right)+\mu_{2}\left[\phi_{2}-E_{r}(i)\right] P_{r}(i) T \\
\text { subject to } & 0 \leq P_{r}(i) T \leq E_{r}(i)
\end{aligned}
$$

Solving problem $\mathcal{P} 4$, we can obtain

Theorem 2: The optimal transmit power of $\mathcal{R}$ in time slot $i$ is given by

$$
P_{r}^{*}(i)= \begin{cases}\frac{E_{r}(i)}{T} & \text { if } E_{r}(i) \geq \phi_{2} \\ {\left[\frac{Q(i)}{\mu_{2} \ln 2\left[\phi_{2}-E_{r}(i)\right]}-\frac{d_{4}^{m} \sigma_{d}^{2}}{\left|h_{2}(i)\right|^{2}}\right]^{\dagger},} & \text { otherwise }\end{cases}
$$

where $[x]^{\dagger}=\min \left\{\max [x, 0], \frac{E_{r}(i)}{T}\right\}$.

Proof: Please refer to Appendix B for the proof.

When the transmit power of $\mathcal{R}$ is allocated as $P_{r}^{*}(i)$, the minimal value of the objective function of problem $\mathcal{P} 4$ is obtained, and denoted by $F_{3}{ }^{*}$. Eq. (30) shows that the transmit power allocated to $\mathcal{R}$ depends on the CSI of the $\mathcal{R}-\mathcal{D}$ link and the BSI of $\mathcal{R}$ only.

As a result, the adaptive joint mode selection and power allocation protocol of the buffer-aided WPCCN is given by

$$
\left\{\begin{array}{lll}
c_{1}(i)=1, & \text { if } & F_{1}^{*}=\min \left\{F_{1}^{*}, F_{2}^{*}, F_{3}^{*}\right\}, \\
c_{2}(i)=1, P_{s}(i)=P_{s}^{*}(i), & \text { if } & F_{2}^{*}=\min \left\{F_{1}^{*}, F_{2}^{*}, F_{3}^{*}\right\}, \\
C_{3}(i)=1, P_{r}(i)=P_{r}^{*}(i), & \text { if } & F_{3}^{*}=\min \left\{F_{1}^{*}, F_{2}^{*}, F_{3}^{*}\right\} .
\end{array}\right.
$$

\subsection{Throughput-delay Tradeoff Analysis}

We note that the data sent by $\mathcal{S}$ is firstly stored in the data buffer of $\mathcal{R}$ until it is scheduled to be transmitted to $\mathcal{D}$, which causes the transmission delay from $\mathcal{S}$ to $\mathcal{D}$ in the buffer-aided JMSPA scheme. In general, the higher throughput is achieved, the larger delay is obtained. Thus, there exits a tradeoff between the throughput and the delay by intuition. In this subsection, we will first present the bound on the time-averaged achievable throughput and the upper bound on the time-averaged data buffer size of relay, and then analyze the time-averaged delay. At the last, we demonstrate the throughput-delay tradeoff achieved by the buffer-aided JMSPA protocol.

For the buffer-aided WPCCN, the optimal time-averaged achievable rate achieved by the joint mode selection and power allocation is denoted by $R^{*}$. Then, we have

Theorem 3: For any $V>0$, there are constants $B \geq 0, \varepsilon \geq 0$ and $\Psi(\varepsilon)$, where $\Psi(\varepsilon)<R^{*}$, then, for some time slot $K$, the time-averaged achievable rate and time-averaged data buffer size achieved by the JMSPA relaying protocol are bounded by 


$$
\begin{gathered}
R^{*}-\frac{B}{V} \leq \limsup _{K \rightarrow \infty} \frac{1}{K} \sum_{i=0}^{K-1} \mathbb{E}\{s(i)\} \leq R^{*} \\
\limsup _{K \rightarrow \infty} \frac{1}{K} \sum_{i=0}^{K-1} \mathbb{E}\{Q(i)\} \leq \frac{B+V\left[R^{*}-\Psi(\varepsilon)\right]}{\varepsilon}
\end{gathered}
$$

Proof: Please refer to Appendix $C$ for the proof.

Eq. (32) suggests that the time-averaged achievable rate increases at the speed of $\mathcal{O}(1 / V)$ and asymptotically converges to the optimal value $R^{*}$ as $V$ increases. Eq. (33) indicates that the time-averaged data queue length is proportional to the value of $V$, i.e., the time-averaged data queue length increases at the speed of $\mathcal{O}(V)$. In other words, Theorem 3 implies that a larger $V$ leads to a larger time-averaged throughput but a larger time-averaged data queue length.

To reveal the throughput-delay tradeoff achieved by the buffer-aided JMSPA relaying protocol, we first present the bound on the time-averaged delay. The time-averaged delay is defined as the ratio between the time-averaged data buffer size and the time-averaged achievable rate. According to Theorem 3, the time-averaged delay is bounded by

$$
\bar{D}=\frac{\frac{1}{K} \sum_{i=0}^{K-1} \mathbb{E}\{Q(i)\}}{\frac{1}{K} \sum_{i=0}^{K-1} \mathbb{E}\{s(i)\}} \leq \frac{B V+V^{2}\left[R^{*}-\Psi(\varepsilon)\right]}{\varepsilon\left[R^{*} V-B\right]}
$$

It can be observed from Eq. (34) that, in the large value region of $V$, where $R^{*} V>>B$, the time-averaged delay is bounded by $\frac{B+V\left[R^{*}-\Psi(\varepsilon)\right]}{\varepsilon R^{*}}$ approximatively. It means that the time-averaged delay increases linearly with $V$, i.e., the time-averaged delay increases at the speed of $\mathcal{O}(V)$. With the aid of system control parameter $V$, the throughput-delay tradeoff can be characterized by $[\mathcal{O}(1 / V), \mathcal{O}(V)]$. Then, the throughput-delay tradeoff can be demonstrated as follows, a larger $V$ leads to a larger time-averaged achievable rate but a larger time-averaged delay.

\subsection{Overhead and Complexity Issues}

In this subsection, we would like to show the overhead and computation complexity of the BSI/CSI based JMSPA protocol by addressing more realization details. In this paper, the proposed buffer-aided JMSPA protocol is implemented in centralized manner. Let us assume the relay node $\mathcal{R}$ is the central node and the channel of each link is reciprocity. Hence, at the beginning of each time slot, the buffer-aided WPCCN may work as follow:

- The relay $\mathcal{R}$ broadcasts the orthogonal pilot signals to the PB, $\mathcal{S}$ and $\mathcal{D}$, thus the PB, $\mathcal{S}$ and $\mathcal{D}$ can drive the CSIs of the $\mathcal{R}-$ PB link, the $\mathcal{R}-\mathcal{S}$ link and the $\mathcal{R}-\mathcal{D}$ link, respectively. In the meanwhile, $\mathcal{S}$ sends the orthogonal pilot signal to the $\mathrm{PB}$, then the PB can estimates the CSI of the $\mathcal{S}-\mathrm{PB}$ link.

- The PB reports the CSIs of the $\mathrm{PB}-\mathcal{S}$ link and the $\mathrm{PB}-\mathcal{R}$ link to central node $\mathcal{R}$ via a dedicated signaling channel. Similarly, $\mathcal{D}$ reports the CSI of the $\mathcal{R}-\mathcal{D}$ link to $\mathcal{R}$ via a dedicated signaling channel. 
- $\mathcal{S}$ reports the CSI of the $\mathcal{S}-\mathcal{R}$ link and its BSI to $\mathcal{R}$ via a dedicated signaling channel. Since $\mathcal{R}$ knows its own BSI, consequently, the CSIs of all links and BSIs of all buffers are gathered at the central node $\mathcal{R}$.

- Based on the obtained CSI and BSI, $\mathcal{R}$ makes a decision which contains the information about which transmission mode is selected and the power allocation based on Theorem $\mathbf{1}$, Theorem 2 and Eq. (31).

- If the WET-EH mode is selected for transmission, $\mathcal{R}$ broadcasts a control message to the $\mathrm{PB}$ and $\mathcal{S}$ and informs the PB to broadcast the energy signal to $\mathcal{S}$ and $\mathcal{R}$; if the WIT-SR mode is selected for transmission, $\mathcal{R}$ broadcasts a control message to inform $\mathcal{S}$ to transmit with the allocated transmit power; if the WIT-RD mode is selected for transmission, $\mathcal{R}$ transmits the information in its data buffer to $\mathcal{D}$ with the assigned transmit power.

According to the above analysis, we can evaluate the signaling overhead of the proposed buffer-aided JMSPA scheme: four orthogonal pilots are required to estimate the CSIs of all links; three control messages are required for the $\mathrm{PB}, \mathcal{S}$ and $\mathcal{D}$ to report the corresponding CSIs and BSI to $\mathcal{R}$ respectively; another two control messages are need for $\mathcal{R}$ to inform the PB and $\mathcal{S}$ the transmission scheduling decisions.

For the implementation of the proposed joint mode selection and power allocation scheme, the most calculations consist in the acquisition of the power allocation scheme for $\mathcal{S}$ and $\mathcal{R}$ by solving the optimization sub-problems $\mathcal{P} 3$ and $\mathcal{P} 4$ within each time slot. In fact, the problem $\mathcal{P} 3$ and $\mathcal{P} 4$ are standard convex problems and exist with closed-form solutions, thus these two problems can be easily solved without using any numerical calculation methods. Therefore, the computation complexity of the proposed buffer-aided JMSPA scheme is very low and can be ignored.

\section{Simulation Results}

In this section, simulation results are presented to evaluate the performance of the proposed buffer-aided relaying scheme and to validate our theoretical analysis. Throughout the simulation, the path loss exponent is set to be $m=2.7$; the energy conversion efficiency of $\mathcal{S}$ and $\mathcal{R}$ is $\eta=0.8$; and the variances of noise in $\mathcal{S}$ and $\mathcal{R}$ are $\sigma_{r}^{2}=\sigma_{d}^{2}=-100 \mathrm{dBm}$, respectively. The length of each time slot is assumed to be $T=1$ and the initial state of the data buffer and energy buffer are both set to be zero. In the simulation, the channel gains of all the links are independent and affected by both the small-scale Rayleigh fading and the large-scale path loss.

In order to evaluate the performance of our proposed buffer-aided relaying scheme, we introduce a relaying scheme without buffer as a performance benchmark, where in each transmit time slot, the first $\alpha T$ time is allocated to the WET stage, the remaining time, $(1-\alpha) T$, is divided into two parts of equal length, and each part is assigned to the $\mathcal{S}-\mathcal{R}$ link and $\mathcal{R}-\mathcal{D}$ link for information transmission, respectively. 


\subsection{The impact of energy harvesting time fraction $\alpha$}

Fig. 3 depicts the impact of energy harvesting time fraction $\alpha$ on the average throughput of the buffer-aided HtT scheme and benchmark scheme (scheme without buffer). In Fig. 3, the transmit power of the PB is $P=1$ Watt, the distance between the PB with $\mathcal{S}$ and $\mathcal{R}$ are $d_{1}=d_{2}=3$ meters and the distance of the $\mathcal{S}-\mathcal{R}$ link and $\mathcal{R}-\mathcal{D}$ link are $d_{3}=d_{4}=5$ meters, respectively.

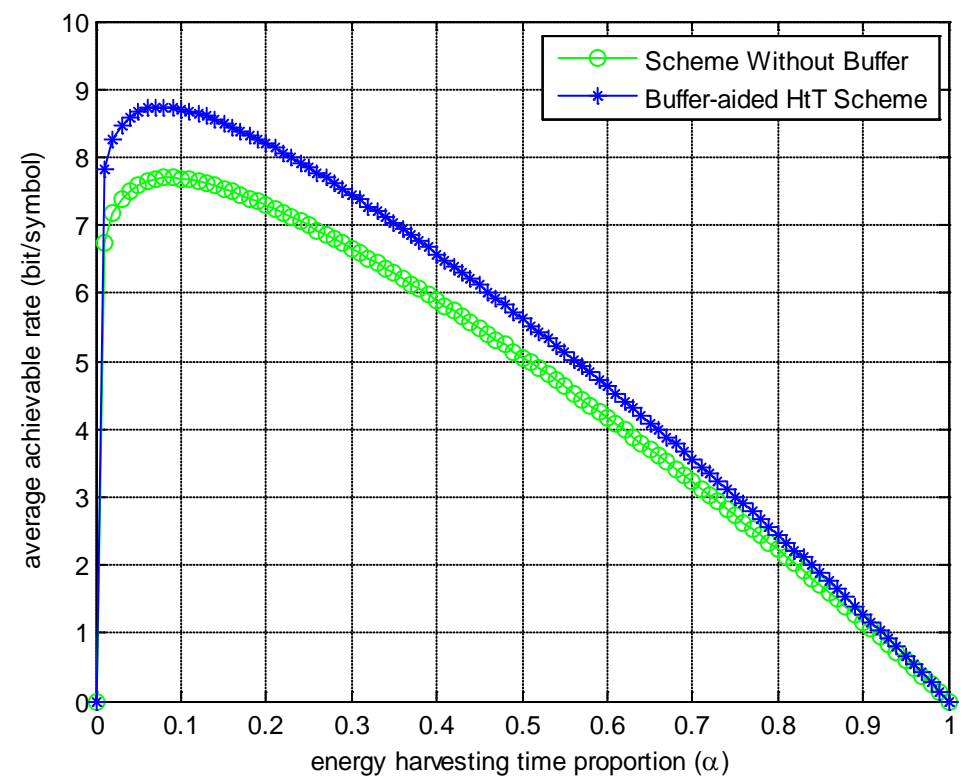

Fig. 3. Average achievable rate VS. $\alpha$.

Fig. 3 shows that, with the energy harvesting time fraction $\alpha$ increasing from 0 to 1 , the average throughput of the buffer-aided HtT scheme and benchmark scheme both increase in the beginning until $\alpha$ reach the optimal value which maximize the throughput of each scheme, and thereafter decrease to zero as $\alpha$ increase from its optimal value to 1 . Especially, when $\alpha$ equals 0 or 1 , the average throughput of both scheme are zero. The concave feature of the throughput performance curves can be explained as follows: When $\alpha$ is equal to 0 , there is no time for $\mathcal{S}$ and $\mathcal{R}$ to harvest energy from the $\mathrm{PB}$, as a result, the transmit power of $\mathcal{S}$ and $\mathcal{R}$ are zero, which leads to the zero throughput; When $\alpha$ increases from 0 to its optimal value, the energy harvested at $\mathcal{S}$ and $\mathcal{R}$ increase, which improves the throughput of the system; As $\alpha$ continues to increase, more and more time are wasted in energy harvesting phase, which gradually reduces the throughput of the system to 0 .

Another observation from Fig. 3 is that throughput of the buffer-aided HtT relaying scheme is always greater than that of the scheme without buffer for any arbitrary $\alpha$. Hence, buffer used in WPCCN can bring a certain throughput gain. 


\subsection{The impact of transmit power of the PB}

For comparison purposes, the average achievable rate of the buffer-aided JMSPA scheme, the buffer-aided HtT scheme and the scheme without buffer is depicted as a function of the transmit power of the power beacon $P$ in Fig. 4, where the distances are set to be $d_{1}=d_{2}=3$ meters and $d_{3}=d_{4}=5$ meters. Specially, for the buffer-aided JMSPA scheme, we set $\mu_{1}=\left(V / \phi_{1}\right)^{2}, \mu_{2}=\left(V / \phi_{2}\right)^{2}, V=100$, and the perturbation values for the energy queue of $\mathcal{S}$ and $\mathcal{R}$ are $\phi_{1}=\phi_{2}=0.001 V+P$.

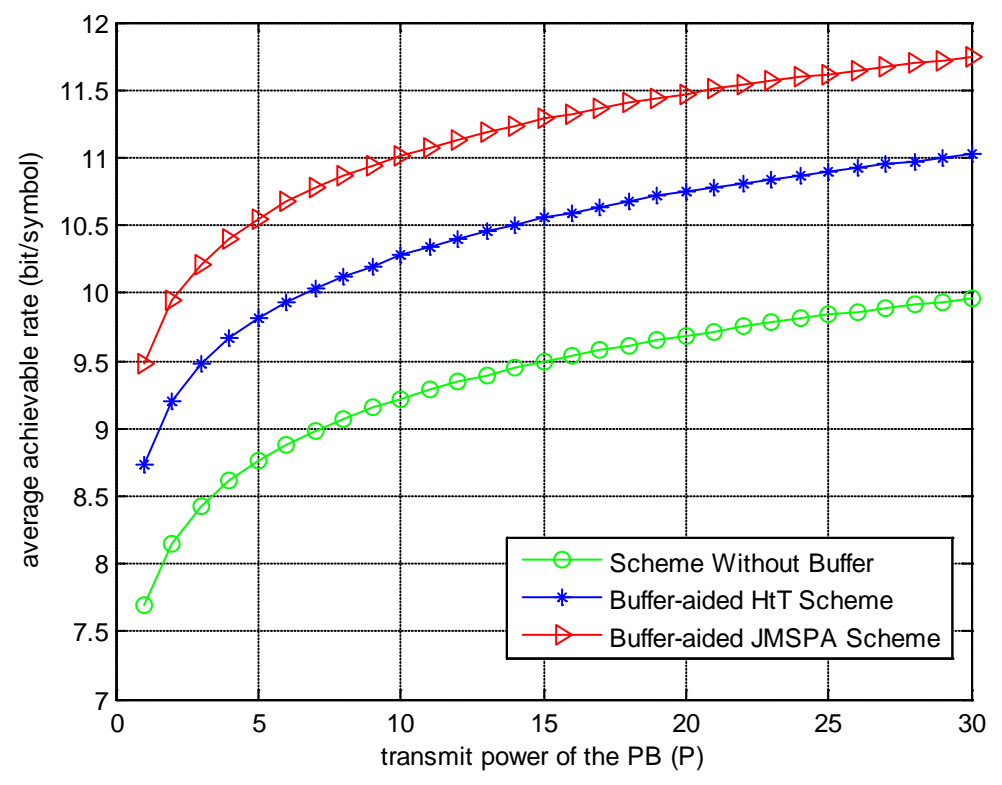

Fig. 4. Average achievable rate VS. transmit power of $\mathrm{PB}(P)$

It can be observed from Fig. 4 that the average achievable rate of three relaying scheme both increase with the transmit power of the PB. This is due to the fact that the larger the transmit power of the $\mathrm{PB}$, the more energy can be harvested at $\mathcal{S}$ and $\mathcal{R}$, then more information can be transmitted from $\mathcal{S}$ to $\mathcal{D}$, which leads to the increasement of the system throughput.

In Fig. 4, we can also see that the throughput performance of the buffer-aided JMSPA scheme outperforms the buffer-aided HtT scheme and the scheme without buffer for any arbitrary $P$. Therefore, we can come to an conclusion that the throughput performance of the buffer-aided WPCCN can be improved further by joint mode selection and power allocation strategy. 


\subsection{The impact of the positions of the PB and $\mathcal{R}$}

In this subsection, we examine the positions of the $\mathrm{PB}$ and the relay node how to affect the throughput performance of the WPCCN. The transmit power of the PB is set to $P=30$ Watt; the overall distance between the PB with $\mathcal{S}$ and $\mathcal{R}$ is fixed at 20 meters, i.e., $d_{1}+d_{2}=20$ meters; similarly, the sum distance of the $\mathcal{S}-\mathcal{R}$ link and $\mathcal{R}-\mathcal{D}$ link is fixed at 20 meters, i.e., $d_{3}+d_{4}=20$ meters. Then, the average achievable rate of three relaying schemes is depicted as a function of $d_{1}$ and $d_{3}$ in Fig. 5, where we set $\mu_{1}=\left(V / \phi_{1}\right)^{2}, \mu_{2}=\left(V / \phi_{2}\right)^{2}$, $\phi_{1}=\eta P^{2} / d_{1}^{m}, \phi_{2}=\eta P^{2} / d_{2}^{m}$ and $V=100$.

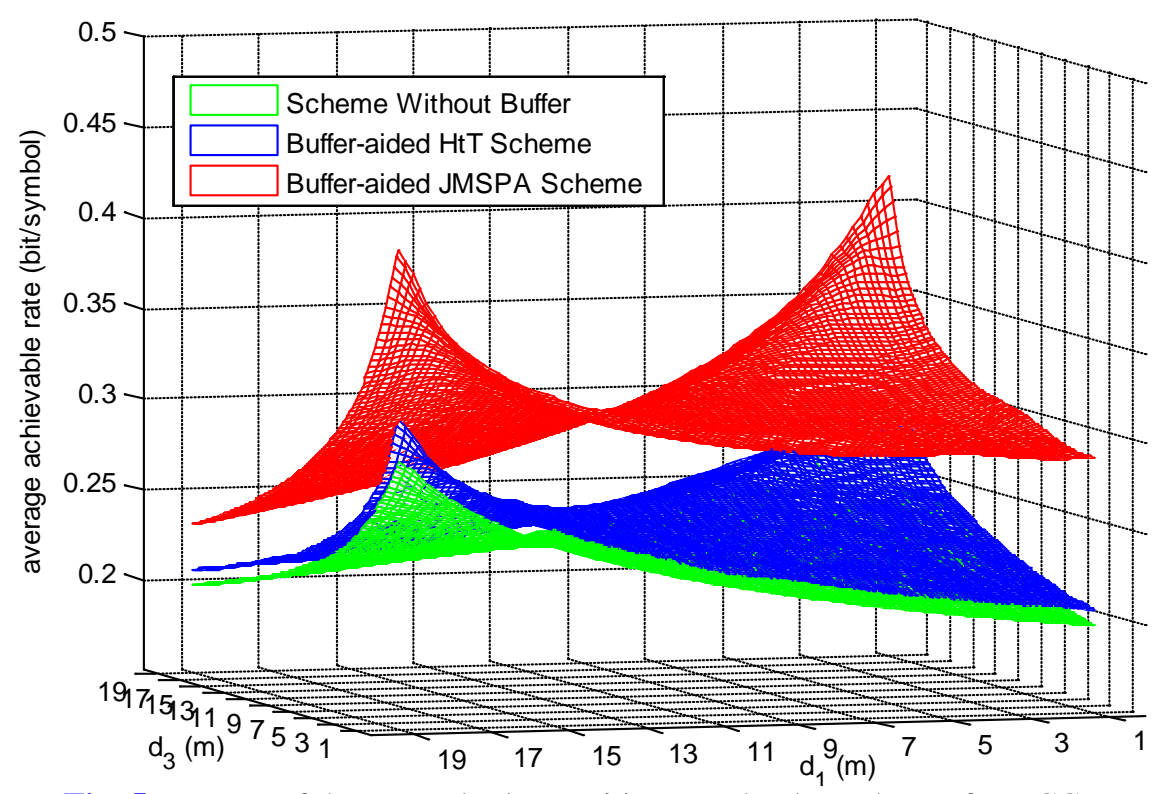

Fig. 5. Impact of the PB and relay positions on the throughput of WPCCN

In Fig. 5, both $d_{1}$ and $d_{3}$ vary from 1 to 19 with a step value of 1 , then, we can observe that the throughput performance of the buffer-aided JMSPA scheme outperforms the buffer-aided HtT scheme and the scheme without buffer for any $d_{1}$ and $d_{3}$. In particular, Fig. 5 shows that for both three relaying schemes, the higher average achievable rate is obtained at the point $\left(d_{1}, d_{3}\right)=(1,19)$, a position where the PB is much more closer to $\mathcal{S}$ than to $\mathcal{R}$, while $\mathcal{R}$ is much more closer to $\mathcal{D}$ than to $\mathcal{S}$; or at the point $\left(d_{1}, d_{3}\right)=(19,1)$, a position where the PB is much closer to $\mathcal{R}$, while $\mathcal{R}$ is much closer to $\mathcal{S}$. On the contrary, the lower average achievable rate is obtained at the point $\left(d_{1}, d_{3}\right)=(1,1)$ or $\left(d_{1}, d_{3}\right)=(19,19)$. For the above results, the reason can be illustrated as that, when the channel gains of all the links are i.i.d, the average achievable rate of the relaying schemes is decided by $\min \left\{1 /\left(d_{1}^{m} d_{3}^{m}\right), 1 /\left(d_{2}^{m} d_{4}^{m}\right)\right\}$, as shown in Eq. (12). Since the maximum value and minimum 
value of $\min \left\{1 /\left(d_{1}^{m} d_{3}^{m}\right), 1 /\left(d_{2}^{m} d_{4}^{m}\right)\right\}$ are $1 /(1 \times 19)^{m}$ and $1 /(19 \times 19)^{m}$ respectively, the maximum and minimum throughput of three relaying schemes are obtained at the point $(1,19)$ and $(19,19)$ respectively.

All in all, we can learn form Fig. 5 that in order to achieve a higher throughput of the considered WPCCN, buffer-aided relaying with joint mode selection and power allocation scheme should be adopted, meanwhile, the PB should be located close to $\mathcal{S}$, while $\mathcal{R}$ should be located close to $\mathcal{D}$; or the PB is located close to $\mathcal{R}$, while $\mathcal{R}$ is located close to $\mathcal{S}$.

\subsection{Validation of analytical results}

For the buffer-aided JMSPA relaying protocol, we first show the simulation results to validate the theoretical analysis of the time-averaged data buffer size and time-averaged delay in Fig. 6. Then the throughput-delay tradeoff achieved by the buffer-aided JMSPA relaying scheme is verified by simulation result in Fig. 7. We set $P=30$ Watt, $d_{1}=d_{2}=3$ meters and $d_{3}=d_{4}=5$ meters. The perturbation values are $\phi_{1}=0.01 \mathrm{~V}+P$ and $\phi_{2}=0.6 \mathrm{~V}+P$, and $\mu_{1}=\left(V / \phi_{1}\right)^{2}, \mu_{2}=\left(V / \phi_{2}\right)^{2}$. Then, the time-averaged data buffer size and time-averaged delay are depicted as a function of $V$ in Fig. 6.
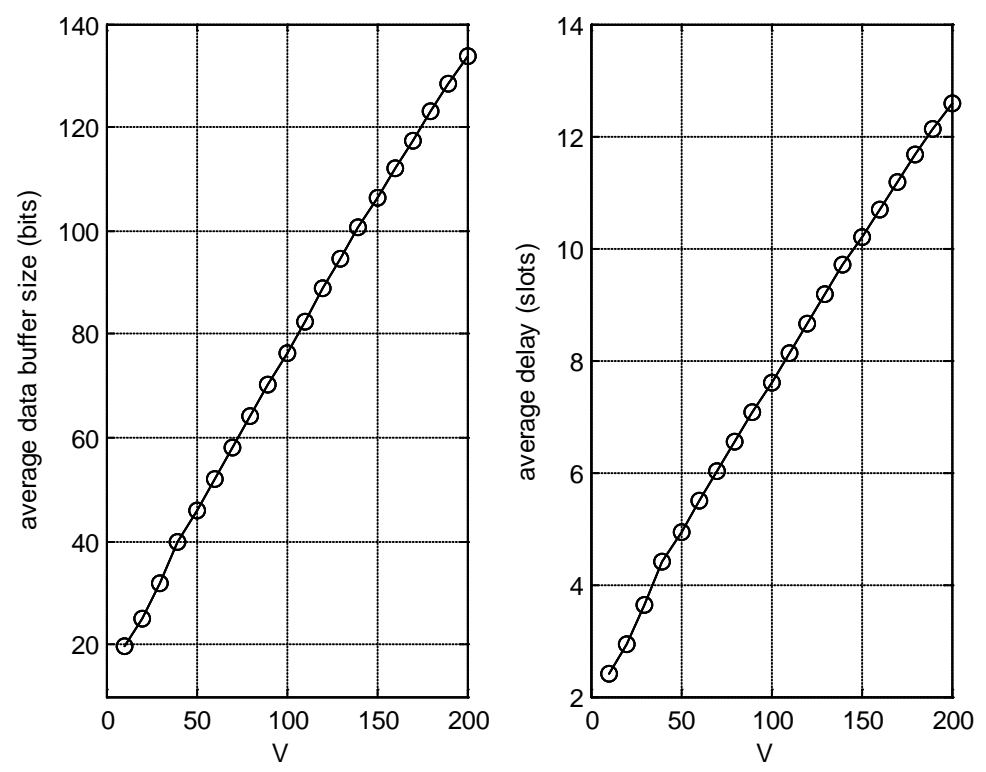

Fig. 6. Average data buffer size and average delay VS. V

It can be observed from Fig. $\mathbf{6}$ that the time-averaged data buffer size and time-averaged delay increase at the speed of $\mathcal{O}(V)$ as the value of $V$ increases from 10 to 200. Especially, in the large region of $V$, both the time-averaged data buffer size and time-averaged delay increase linearly with $V$, which validate the analysis in Theorem 3. 
The time-averaged delay is depicted as a function of average achievable rate in Fig. 7.

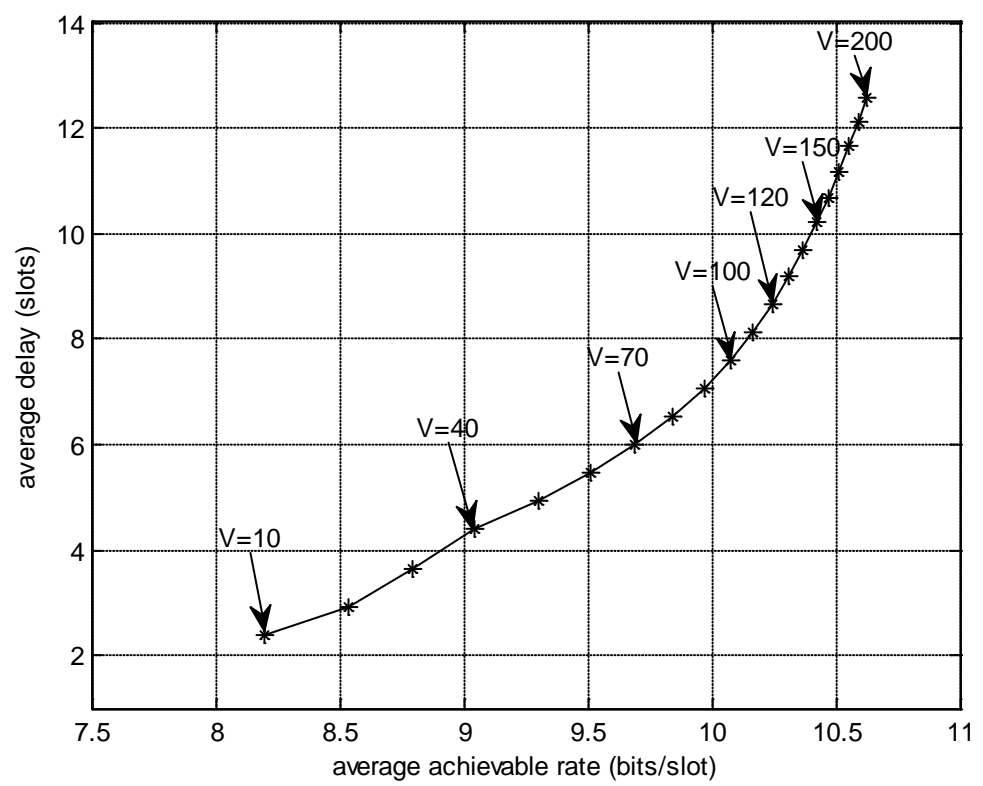

Fig. 7. Average delay VS. average achievable rate

Fig. 7 shows that for the buffer-aided WPCCN with JMSPA scheme, the larger the time-averaged delay, the higher time-averaged throughput is obtained, which presents a throughput-delay tradeoff of the buffer-aided JMSPA protocol. In practice, the throughput-delay tradeoff curve can be applied to guide the system design by adjusting the control parameter $V$. For instance, if the system is delay-constrained, then a small $V$ should be chosen. In contrast, if the system pays more attention to the throughput, then a larger $V$ is required.

\section{Conclusion and Future Works}

In this paper, we have investigated a buffer-aided WPCCN which consists of $\mathcal{S}, \mathcal{R}, \mathcal{D}$, and a dedicated PB. Considering the buffer-aided WPCCN, we proposed two relaying protocols, namely, the buffer-aided HtT protocol and the buffer-aided JMSPA protocol, respectively. For the buffer-aided HtT protocol, we derived the expression of the time-averaged achievable rate in closed form. For the buffer-aided JMSPA protocol, maximizing the time-averaged throughput was formulated as a stochastic optimal problem. We solved the optimal problem by employing Lyapunov optimization theory and obtained the optimal mode selection scheme and power allocation scheme. In addition, we derived the theoretical bounds on the time-averaged achievable rate, time-averaged data buffer size and time-averaged delay, then revealed the throughput-delay tradeoff achieved by the buffer-aided JMSPA protocol. The simulation results showed that the proposed buffer-aided relaying protocol can improve the throughput performance of WPCCN and validated the theoretical analysis. 
In realistic network, acquisition of the ideal CSI/BSI can be challenging. For instance, the estimation error attached to the channel estimation methods would results in an imperfect CSI. Moreover, the obtained CSI/BSI by estimation or feedback is always outdated in a fast time varying scenario. For a practical buffer-aided network with imperfect or outdated CSI/BSI, a robust adaptive relaying protocol, which can approach the throughput performance bound that our JMSPA protocol achieved in this paper, will be left for investigation in future work.

\section{Appendix}

\section{A. Proof of Theorem 1}

We define the objective function in problem $\mathcal{P} 3$ as $F_{2}$, then if $Q(i) \geq V$ and $\phi_{1} \geq E_{s}(i), F_{2}$ is a monotone increasing function of $P_{s}(i)$. Thus, when $P_{s}(i)=0, F_{2}$ obtains the minimal value. If $Q(i)<V$ and $\phi_{1}<E_{s}(i), F_{2}$ is a monotone decreasing function of $P_{s}(i)$, the minimal value of $F_{2}$ is achieved when $P_{s}(i)=E_{s}(i) / T$.

In addition to the two cases above, $F_{2}$ is not a monotone function of $P_{s}(i)$. We first calculate the stationary point of $F_{2}$, which is obtained as

$$
P_{s}(i)=\frac{Q(i)-V}{\mu_{1} \ln 2\left[E_{s}(i)-\phi_{1}\right]}-\frac{d_{3}^{m} \sigma_{r}^{2}}{\left|h_{1}(i)\right|^{2}}
$$

Taking the second-order differential of $F_{2}$ with respect to $P_{s}(i)$ yields

$$
F_{2}^{\prime \prime}=-\frac{Q(i)-V}{\ln 2} \cdot \frac{\left|h_{1}(i)\right|^{4} T}{\left[d_{3}^{m} \sigma_{r}^{2}+P_{s}(i)\left|h_{1}(i)\right|^{2}\right]^{2}}
$$

Hence, if $Q(i)<V$, then $F_{2}^{\prime \prime}>0, F_{2}$ is a convex function. As a result, if the stationary point is located in the region of $\left[0, E_{s}(i) / T\right]$, the minimal value of $F_{2}$ can be obtained at the stationary point, otherwise, the minimal value will be achieved at the endpoints, i.e., 0 or $E_{s}(i) / T$.

If $Q(i)>V$, then $F_{2}^{\prime \prime}<0, F_{2}$ is a concave function. In this case, the minimal value of $F_{2}$ is obtained at the endpoints. We define $\Delta=F_{2}\left(E_{s}(i) / T\right)-F_{2}(0)$. Therefore, if $\Delta \geq 0$, the minimal value of $F_{2}$ is achieved at $P_{s}(i)=0$, otherwise the minimal value of $F_{2}$ is obtained at $P_{s}(i)=E_{s}(i) / T$.

This concludes the proof of Theorem 1.

\section{B. Proof of Theorem 2}

Firstly, we define the objective function in problem $\mathcal{P} 4$ as $F_{3}$. If $\phi_{2} \leq E_{r}(i), F_{3}$ monotonously decreases with $P_{r}(i)$, obviously $F_{3}$ achieves the minimal value at $P_{r}(i)=E_{r}(i) / T$. Otherwise $\phi_{2}>E_{r}(i), F_{3}$ is a convex function. Differentiating $F_{3}$ with respect to $P_{r}(i)$ and equating the result to zero, the unique stationary point is calculated as 


$$
P_{r}(i)=\frac{Q(i)}{\mu_{2} \ln 2\left[\phi_{2}-E_{r}(i)\right]}-\frac{d_{4}^{m} \sigma_{d}^{2}}{\left|h_{2}(i)\right|^{2}}
$$

Since $F_{3}$ is convex, then if the stationary point in Eq. (37) is located in the region of [0, $\left.E_{r}(i) / T\right]$, the minimal value of $F_{3}$ could be achieved at the stationary point. Otherwise, the minimal value will be obtained at the endpoints, i.e., 0 or $E_{r}(i) / T$.

To sum up, when the WIT-RD mode is selected for transmission, the optimal transmit power of $\mathcal{R}$ is presented by

$$
P_{r}^{*}(i)= \begin{cases}\frac{E_{r}(i)}{T} & \text { if } E_{r}(i) \geq \phi_{2} \\ {\left[\frac{Q(i)}{\mu_{2} \ln 2\left[\phi_{2}-E_{r}(i)\right]}-\frac{d_{4}^{m} \sigma_{d}^{2}}{\left|h_{2}(i)\right|^{2}}\right]^{\dagger},} & \text { otherwise }\end{cases}
$$

where $[x]^{\dagger}=\min \left\{\max [x, 0], \frac{E_{r}(i)}{T}\right\}$.

This completes the proof of Theorem 2 .

\section{Proof of Theorem 3}

According to Slater Condition [32], for any $V>0$, there are constants $\varepsilon>0$ and $\Psi(\varepsilon)$ that satisfies

$$
\mathbb{E}\{L(i+1)-L(i) \mid \mathbf{Z}(i)\}-V \mathbb{E}\{s(i) \mid \mathbf{Z}(i)\} \leq B-V \Psi(\varepsilon)-\varepsilon Q(i)
$$

Taking expectations of (39) in both sides yields

$$
\mathbb{E}\{L(i+1)\}-\mathbb{E}\{L(i)\}-V \mathbb{E}\{s(i)\} \leq B-V \Psi(\varepsilon)-\varepsilon \mathbb{E}\{Q(i)\}
$$

Summing (40) over $i \in\{0,1, \cdots, K-1\}$ for some time slot $K>0$, we can obtain

$$
\mathbb{E}\{L(K)\}-\mathbb{E}\{L(0)\}-V \sum_{i=0}^{K-1} \mathbb{E}\{s(i)\} \leq K B-K V \Psi(\varepsilon)-\varepsilon \sum_{i=0}^{K-1} \mathbb{E}\{Q(i)\}
$$

Rearranging terms in the above and considering the fact that $L(K) \geq 0$ yields

$$
\varepsilon \sum_{i=0}^{K-1} \mathbb{E}\{Q(i)\} \leq K B-K V \Psi(\varepsilon)+V \sum_{i=0}^{K-1} \mathbb{E}\{s(i)\}+\mathbb{E}\{L(0)\}
$$

Dividing both sides of (42) by $\varepsilon K$ shows that

$$
\frac{1}{K} \sum_{i=0}^{K-1} \mathbb{E}\{Q(i)\} \leq \frac{B+V\left[\sum_{i=0}^{K-1} \mathbb{E}\{s(i)\}-\Psi(\varepsilon)\right]}{\varepsilon}+\frac{\mathbb{E}\{L(0)\}}{\varepsilon K}
$$

Taking a supremum limit as $K \rightarrow \infty$ and combining with $\limsup _{K \rightarrow \infty} \frac{1}{K} \sum_{i=0}^{K-1} \mathbb{E}\{s(i)\} \leq R^{*}$ yields

$$
\limsup _{K \rightarrow \infty} \frac{1}{K} \sum_{i=0}^{K-1} \mathbb{E}\{Q(i)\} \leq \frac{B+V\left[R^{*}-\Psi(\varepsilon)\right]}{\varepsilon}
$$

Thus, we obtain the upper bound on the average data buffer size. 
Similarly, we have

$$
\mathbb{E}\{L(i+1)-L(i) \mid \mathbf{Z}(i)\}-V \mathbb{E}\{s(i) \mid \mathbf{Z}(i)\} \leq B-V R^{*}-\varepsilon Q(i)
$$

Taking expectations at both sides, summing the telescoping items, and rearranging terms yields

$$
V \sum_{i=0}^{K-1} \mathbb{E}\{s(i)\} \geq \mathbb{E}\{L(K)\}-\mathbb{E}\{L(0)\}+\varepsilon \sum_{i=0}^{K-1} \mathbb{E}\{Q(i)\}-K B+K V R^{*}
$$

Considering $L(K) \geq 0$ and $\varepsilon \sum_{i=0}^{K-1} \mathbb{E}\{Q(i)\} \geq 0$, we have

$$
V \sum_{i=0}^{K-1} \mathbb{E}\{s(i)\} \geq K V R^{*}-K B-\mathbb{E}\{L(0)\}
$$

Dividing (47) by $K V$ and taking a limit as $K \rightarrow \infty$ yields

$$
\limsup _{K \rightarrow \infty} \frac{1}{K} \sum_{i=0}^{K-1} \mathbb{E}\{s(i)\} \geq R^{*}-\frac{B}{V}
$$

As a result, we have

$$
R^{*}-\frac{B}{V} \leq \limsup _{K \rightarrow \infty} \frac{1}{K} \sum_{i=0}^{K-1} \mathbb{E}\{s(i)\} \leq R^{*}
$$

This concludes the proof.

\section{References}

[1] M.-L. Ku, W. Li, Y. Chen, and K. R. Liu, “Advances in energy harvesting communications: past, present, and future challenges,” IEEE Commun. Surveys Tuts., vol. 18, no. 2, pp. 1384-1412, Second Quarter 2016. Article (CrossRef Link)

[2] X. Lu, P. Wang, D. Niyato, D. I. Kim, and Z. Han, "Wireless networks with RF energy harvesting: a contemporary survey,” IEEE Commun. Surveys Tuts., vol. 17, no. 2, pp. 757-789, Second Quarter 2015. Article (CrossRef Link)

[3] S. Bi, C. Ho, and R. Zhang, "Wireless powered communication: opportunities and challenges," IEEE Commun. Mag., vol. 53, no. 4, pp. 117-125, April, 2015. Article (CrossRef Link)

[4] S. Bi, Y. Zeng, and R. Zhang, "Wireless powered communication networks: an overview," IEEE Wireless Commun., vol. 23, no. 2, pp. 10-18, Apr. 2016. Article (CrossRef Link)

[5] K. Huang, C. Zhong, and G. Zhu, "Some new research trends in wirelessly powered communications,” IEEE Wireless Commun., vol. 23, no. 2, pp. 19-27, Apr. 2016. Article (CrossRef Link)

[6] H. Gao, W. Ejaz, and M. Jo, "Cooperative wireless energy harvesting and spectrum sharing in 5G networks,” IEEE Access, vol. 4, pp. 3647-3658, Jul. 2016. Article (CrossRef Link)

[7] F. Zhao, L. Wei, and H. Chen, "Optimal time allocation for wireless information and power transfer in wireless powered communication systems,” IEEE Trans. Veh. Tech., vol. 65, no. 3, pp. 1830-1835, Mar. 2016. Article (CrossRef Link)

[8] J. Yang, Q. Yang, K. S. Kwak, and R. R. Rao, "Power-delay tradeoff in wireless powered communication networks,” IEEE Trans. Veh. Tech., vol. 66, no. 4, pp. 3280-3292, Apr. 2017. Article (CrossRef Link) 
[9] H. Ju and R. Zhang, “Throughput maximization in wireless powered communication networks," IEEE Trans. Wireless Commun., vol. 13, no. 1, pp. 418-428, Jan. 2014. Article (CrossRef Link)

[10] H. Lee, K.-J. Lee, H. Kim, B. Clerckx, and I. Lee, "Resource allocation techniques for wireless powered communication networks with energy storage constraint," IEEE Trans. Wireless Commun., vol. 15, no. 4, pp. 2619-2628, Apr. 2016. Article (CrossRef Link)

[11] I. Krikidis, S. Timotheou, S. Nikolaou, G. Zheng, D. W. K. Ng, and R. Schober, "Simultaneous wireless information and power transfer in modern communication systems," IEEE Commun. Mag., vol. 52, no. 11, pp. 104-110, Nov. 2014. Article (CrossRef Link)

[12] R. Zhang and C. K. Ho, "MIMO broadcasting for simultaneous wireless information and power transfer,” IEEE Trans. Wireless Commun., vol. 12, no. 5, pp. 1989-2001, May 2013. Article (CrossRef Link)

[13] Q. Shi, L. Liu, W. Xu, and R. Zhang, "Joint transmit beamforming and receive power splitting for MISO SWIPT systems,” IEEE Trans. Wireless Commun., vol. 13, no. 6, pp. 3269-3280, Jun. 2014. Article (CrossRef Link)

[14] M. R. Khandaker and K.-K. Wong, "SWIPT in MISO multicasting systems," IEEE Commun. Lett., vol. 3, no. 3, pp. 277-280, Jun. 2014. Article (CrossRef Link)

[15] Y. Liu, Z. Ding, M. Elkashlan, and H. V. Poor, "Cooperative non-orthogonal multiple access with simultaneous wireless information and power transfer,” IEEE J. Sel. Areas Commun., vol. 34, no. 4, pp. 938-953, Apr. 2016. Article (CrossRef Link)

[16] A. A. Nasir, X. Zhou, S. Durrani, and R. A. Kennedy, "Relaying protocols for wireless energy harvesting and information processing," IEEE Trans. Wireless Commun., vol. 12, no. 7, pp. 3622-3636, Jul. 2013. Article (CrossRef Link)

[17] A. A. Nasir, X. Zhou, S. Durrani, and R. A. Kennedy, "Throughput and ergodic capacity of wireless energy harvesting based DF relaying network,” in Proc. of IEEE Inf. Conf. Commun., 2014, pp. 4066-4071. Article (CrossRef Link)

[18] Y. Gu and S. A" 1ssa, "RF-based energy harvesting in decode-and-forward relaying systems: ergodic and outage capacities," IEEE Trans. Wireless Commun., vol. 14, no. 11, pp. 6425-6434, Nov. 2015. Article (CrossRef Link)

[19] Y. Cai, M. M. Zhao, Q. Shi, B. Champagne, and M. J. Zhao, “Joint transceiver design algorithms for multiuser MISO relay systems with energy harvesting,” IEEE Trans. Commun., vol. 64, no. 10, pp. 4147-4164, Oct. 2016. Article (CrossRef Link)

[20] Y. Zeng and R. Zhang, "Full-duplex wireless-powered relay with self-energy recycling," IEEE Wireless Commun. Lett., vol. 4, no. 2, pp. 201-204, Apr. 2015. Article (CrossRef Link)

[21] L. Zhang, Y. Cai, M. Zhao, B. Champagne, and L. Hanzo, "Nonlinear MIMO transceivers improve wireless-powered and self-interference-aided relaying," IEEE Trans. Wireless Commun., vol. 16, no. 10, pp. 6953-6966, Oct. 2017. Article (CrossRef Link)

[22] N. Zlatanov, A. Ikhlef, T. Islam, and R. Schober, "Buffer-aided cooperative communications: opportunities and challenges,” IEEE Commun. Mag., vol. 52, no. 4, pp. 146-153, Apr. 2014. Article (CrossRef Link)

[23] N. Zlatanov, R. Schober, and P. Popovski, "Buffer-aided relaying with adaptive link selection," IEEE J. Sel. Areas Commun., vol. 31, no. 8, pp. 1530-1542, Aug. 2013. Article (CrossRef Link)

[24] N. Zlatanov and R. Schober, "Buffer-aided relaying with adaptive link selection--fixed and mixed rate transmission,” IEEE Trans. Inf. Theory, vol. 59, no. 5, pp. 2816-2840, May 2013. Article (CrossRef Link)

[25] V. Jamali, N. Zlatanov, H. Shoukry, and R. Schober, “Achievable rate of the half-duplex multi-hop buffer-aided relay channel with block fading,” IEEE Trans. Wireless Commun., vol. 14, no. 11, pp. 6240-6256, Nov. 2015. Article (CrossRef Link)

[26] V. Jamali, N. Zlatanov, A. Ikhlef, and R. Schober, “Adaptive mode selection in bidirectional buffer-aided relay networks with fixed transmit powers," in Proc. of Euro. Sig. Proces. Conf.(EUSIPCO), Sept. 2013. Article (CrossRef Link)

[27] V. Jamali, N. Zlatanov, and R. Schober, "Bidirectional buffer-aided relay networks with fixed rate transmission--part i: Delay-unconstrained case,” IEEE Trans. Wireless Commun., vol. 14, no. 3, pp. 1323-1338, Mar. 2015. Article (CrossRef Link) 
[28] V. Jamali, N. Zlatanov, and R. Schober, "Bidirectional buffer-aided relay networks with fixed rate transmission--part ii: Delay-constrained case,” IEEE Trans. Wireless Commun., vol. 14, no. 3, pp. 1339-1355, Mar. 2015. Article (CrossRef Link)

[29] I. Krikidis, T. Charalambous, and J. S. Thompson, "Buffer-aided relay selection for cooperative diversity systems without delay constraints,” IEEE Trans. Wireless Commun., vol. 11, no. 5, pp. 1957-1967, May 2012. Article (CrossRef Link)

[30] A. Ikhlef, D. S. Michalopoulos, and R. Schober, "Max-max relay selection for relays with buffers," IEEE Trans. Wireless Commun., vol. 11, no. 3, pp. 1124-1135, Mar. 2012.

Article (CrossRef Link)

[31] N. Zlatanov, V. Jamali, and R. Schober, “Achievable rates for the fading half-duplex single relay selection network using buffer-aided relaying,” IEEE Trans. Wireless Commun., vol. 14, no. 8, pp. 4494-4507, Aug. 2015. Article (CrossRef Link)

[32] M. Neely, Stochastic Network Optimization with Application to Communication and Queueing Systems. San Rafael, CA, USA: Morgan \& Claypool, 2010. Article (CrossRef Link)

[33] L. Huang and M. J. Neely, "Utility optimal scheduling in energy-harvesting networks," IEEE/ACM Trans. Netw., vol. 21, no. 4, pp. 1117-1130, Aug. 2013. Article (CrossRef Link)

[34] C. Zhong, G. Zheng, Z. Zhang, and G. K. Karagiannidis, “Optimum wirelessly powered relaying,” IEEE Signal Process. Lett., vol. 22, no. 10, pp. 1728-1732, Oct. 2015. Article (CrossRef Link)

[35] B. Xia, Y. Fan, J. Thompson, and H. V. Poor, "Buffering in a three-node relay network," IEEE Trans. Wireless Commun., vol. 7, no. 11, pp. 4492-4496, Nov. 2008. Article (CrossRef Link)

[36] N. Nomikos, T. Charalambous, I. Krikidis, and D. N. Skoutas, "A survey on buffer-aided relay selection,” IEEE Commun. Surveys Tuts., vol. 18, no. 2, pp. 1073-1097, Second Quarter 2016. Article (CrossRef Link)

[37] S. Boyd and L. Vandenberghe, Convex optimization. Cambridge, U.K.: Cambridge Univ. Press, 2004. Article (CrossRef Link) 


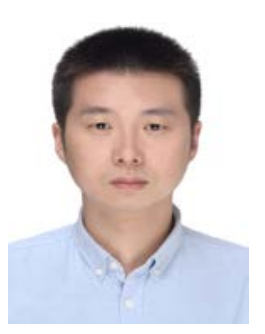

Jun Zhan received the B.E. degree in electronic and information engineering from Three Gorges University, China, in 2011. He is currently pursuing the Ph.D. degree at the School of Information Science and Technology in Southwest Jiaotong University, Chengdu, China. His current research interests include the design of relaying protocol for buffer-aided cooperative networks and energy harvesting wireless communication.

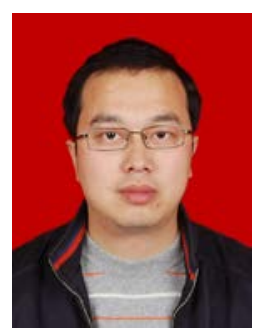

Xiaohu Tang received the B.S. degree in applied mathematics from Northwest Polytechnic University, Xi'an, China, in 1992, the M.S. degree in applied mathematics from Sichuan University, Chengdu, China, in 1995, and the Ph.D. degree in electronic engineering from Southwest Jiaotong University, Chengdu, in 2001. From 2003 to 2004, he was a Research Associate with the Department of Electrical and Electronic Engineering, The Hong Kong University of Science and Technology. From 2007 to 2008, he was a Visiting Professor with the University of Ulm, Germany. Since 2001, he has been with the School of Information Science and Technology, Southwest Jiaotong University, where he is currently a Professor. His current research interests include coding theory, network security, distributed storage, and information processing for big data. Dr. Tang was a recipient of the National Excellent Doctoral Dissertation Award in 2003 (China), the Humboldt Research Fellowship in 2007 (Germany), and the Outstanding Young Scientist Award by NSFC in 2013 (China). He serves as an Associate Editor for several journals, including the IEEE TRANSACTIONS ON INFORMATION THEORY and IEICE Transactions on Fundamentals, and has served on a number of technical program committees of conferences.

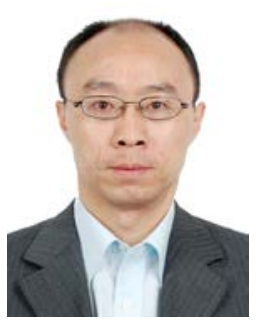

Qingchun Chen received the B.Sc. degree and the M.Sc. degree (Hons.) from Chongqing University, China, in 1994 and 1997, respectively, and the Ph.D. degree from Southwest Jiaotong University, China, in 2004. He joined Southwest Jiaotong University since 2004 as an Associate Professor and then as a Full Professor since 2009. He has authored and co-authored over 100 research papers, two book chapters, and 40 patents. His research interest includes wireless communication, wireless network, channel coding, and signal processing. He received the 2016 IEEE GLOBECOM Best Paper Award. He served as an Associate Editor of the IEEE ACCESS (2015-present). 